\title{
Early surgery for superficial supratentorial spontaneous intracerebral hemorrhage: a Finnish Intensive Care Consortium study
}

\author{
Teemu Luostarinen ${ }^{1}$ D $\cdot$ Jarno Satopää ${ }^{2} \cdot$ Markus B Skrifvars $^{3} \cdot$ Matti Reinikainen $^{4} \cdot$ Stepani Bendel $^{4} \cdot$ Sami Curtze $^{5}$. \\ Gerli Sibolt $^{5} \cdot$ Nicolas Martinez-Majander $^{5} \cdot$ Rahul Raj $^{2}$
}

Received: 28 April 2020 / Accepted: 18 June 2020 / Published online: 29 June 2020

(C) The Author(s) 2020

\begin{abstract}
Background The benefits of early surgery in cases of superficial supratentorial spontaneous intracerebral hemorrhage (ICH) are unclear. This study aimed to assess the association between early ICH surgery and outcome, as well as the cost-effectiveness of early ICH surgery.

Methods We conducted a retrospective, register-based multicenter study that included all patients who had been treated for supratentorial spontaneous ICH in four tertiary intensive care units in Finland between 2003 and 2013. To be included, patients needed to have experienced supratentorial ICHs that were $10-100 \mathrm{~cm}^{3}$ and located within $10 \mathrm{~mm}$ of the cortex. We used a multivariable analysis, adjusting for the severity of the illness and the probability of surgical treatment, to assess the independent association between early ICH surgery ( $\leq 1$ day), 12-month mortality rates, and the probability of survival without permanent disability. In addition, we assessed the cost-effectiveness of ICH surgery by examining the effective cost per 1-year survivor (ECPS) and per independent survivor (ECPIS).

Results Of 254 patients, $27 \%$ were in the early surgery group. Overall 12-month mortality was $39 \%$, while $29 \%$ survived without a permanent disability. According to our multivariable analysis, early ICH surgery was associated with lower 12-month mortality rates (odds ratio [OR] $0.22,95 \%$ confidence intervals [CI] 0.10-0.51), but not with a higher probability of survival without permanent disability (OR 1.23, 95\% CI 0.59-2.56). For the early surgical group, the ECPS and ECPIS were $€ 111,409$ and $€ 334,227$, respectively. For the non-surgical cohort, the ECPS and ECPIS were $€ 76,074$ and $€ 141,471$, respectively.

Conclusions Early surgery for superficial ICH is associated with a lower 12-month mortality risk but not with a higher probability of survival without a permanent disability. Further, costs were higher and cost-effectiveness was, thus, worse for the early surgical cohort.
\end{abstract}

Keywords Intracerebral hemorrhage $\cdot$ Stroke $\cdot$ Surgery $\cdot$ Neurosurgery $\cdot$ Outcome $\cdot$ Mortality

This article is part of the Topical Collection on Neurosurgery general

Electronic supplementary material The online version of this article (https://doi.org/10.1007/s00701-020-04470-y) contains supplementary material, which is available to authorized users.

Teemu Luostarinen

teemu.luostarinen@hus.fi

1 Division of Anesthesiology, Department of Anesthesiology, Intensive Care, and Pain Medicine, Helsinki University Hospital and University of Helsinki, Topeliuksenkatu 5, PO BOX 266, 00029 HUS Helsinki, Finland

2 Department of Neurosurgery, Helsinki University Hospital and University of Helsinki, Helsinki, Finland
3 Department of Emergency Care and Services, Helsinki University Hospital and University of Helsinki, Helsinki, Finland

4 Department of Anesthesiology and Intensive Care, University of Eastern Finland and Kuopio University Hospital, Kuopio, Finland

5 Department of Neurology, Helsinki University Hospital and University of Helsinki, Helsinki, Finland 


\section{Introduction}

Spontaneous intracerebral hemorrhage (ICH) is associated with high mortality and morbidity rates [15]. It is a great burden on healthcare systems, and the associated treatment costs are high because it requires an extended rehabilitation period $[17,20]$. Despite there being multiple randomized studies into the issue, it is still unclear what the benefits are to surgery being carried out during the early stages of ICH treatment $[13,14,23]$.

Using data on ICH patients that were available in a large, retrospective multicenter intensive care unit (ICU) database, we assessed the association between early ICH surgery and mortality rates after spontaneous supratentorial ICH, using the same inclusion criteria that were used for STICH II [14]. We hypothesized that when compared to non-surgical treatment, early surgery would be associated with lower mortality rates but not with an increased rate of survival without permanent disability. A secondary aim was to assess the costeffectiveness of surgical treatment for ICH. Our hypothesis was that early ICH surgery would be associated with lower cost-effectiveness per 1-year survivor but with increased costeffectiveness per independent survivor.

\section{Methods}

\section{Patient selection and variables}

We conducted a retrospective, multicenter study using a highquality ICU database from the Finnish Intensive Care Consortium (FICC). FICC includes prospectively collected data from most of the ICUs in Finland, with the exception of some specialized units. FICC has previously been described in detail [18]. Data on patients in FICC were identified based on their ICU admission diagnosis, after which the diagnoses were manually verified. We included adult patients ( $\geq 18$ years) who had been treated between January 2003 and December 2013 for a spontaneous ICH in any of four tertiary ICUs in Finland (providing neurointensive and neurosurgical care). In Finland, there are five university hospitals that provide neurointensive and neurosurgical care, of which four provided data to FICC during the study period. We only included patients with supratentorial ICHs that were located $\leq 10 \mathrm{~mm}$ from the cortex and were between 10 and $100 \mathrm{~cm}^{3}$ in size [14]. We excluded readmitted patients and patients with missing Glasgow Coma Scale (GCS) scores.

Three authors who had been blinded to information about the patients' treatments or outcomes evaluated all of the patients' computed tomography (CT) scans [3]. Hematoma volume was measured using the $\mathrm{ABC} / 2$ method [9]. Midline shift was measured in millimeters. Intraventricular hemorrhage
$(\mathrm{IVH})$ in any ventricle was noted. Depth from the cortex was measured in millimeters, from the point closest to the cortex.

Patients in the medical treatment group were treated according to general neurointensive care principles and local guidelines, which are based on the ESO and AHA guidelines $[6,19]$.

\section{Outcome and cost variables}

Our primary outcome was 12 -month mortality rates. The dates of death for all patients were obtained from Statistics Finland. We also report ICU, hospital, and 30-day mortality rates. As a secondary outcome, we used a surrogate variable of survival without permanent disability, with a permanent disability defined as a disability for which the patient was granted a permanent disability allowance or disability pension by the Social Insurance Institute (Kela) in Finland. Kela is a Finnish government agency that is funded by taxes, insurance payments, and municipalities and that provides all social security payments for the country. In order to qualify for a permanent disability allowance or disability pension, Kela mandates that a person must be unable to independently carry out daily activities (e.g., self-hygiene, basic housekeeping, taking care of things outside the home) or to be unable to return to work for a minimum of one consecutive year. We therefore defined all patients who were alive 1 year after ICU admission and who had been granted a permanent disability allowance or pension by Kela (by September 30, 2016) as permanently disabled [16].

The total healthcare cost variable included the index university hospital treatment costs, rehabilitation hospital costs, and social security costs up to 1 year after admission. The cost variables have previously been described in detail $[2,16,17]$. We adjusted all costs according to the average annual Consumer Price Index in Finland into euros, based on the exchange rate in 2019 (data from Statistics Finland):

CPI adjusted costs $=$ Costs $\times \frac{\text { CPI in } 2019}{\text { Admission year CPI }}$

\section{Statistical analyses}

We used SPSS Statistics 24.0 for Mac (IBM Corp, Armonk, NY) and Stata Statistical Software for Mac (StataCorp LP, College Station, TX) to carry out statistical analyses. We used a $\chi^{2}$ test to compare categorical data (and a Bonferroni correction when appropriate) and presented the results as percentages $(\%)$. We tested continuous data for normality and found that all continuous variables were highly skewed. Thus, we used non-parametric Mann-Whitney $U$ and Kruskal-Wallis tests for continuous data between groups. Continuous data 
are presented as medians with interquartile ranges. We considered $p$ values $<0.05$ as statistically significant.

To assess the independent association between early ICH surgery and outcomes, we used multivariable logistic regression modeling, adjusting for the severity of illness and likelihood of surgical treatment (primary analysis). We adjusted for severity of illness by creating a severity of illness model that included variables that significantly associated with 12-month mortality in univariate analysis, as well as prognostic factors from our recent study [3]. Thus, the final severity of illness model included the following: age (continuous), GCS score (defined as the worst score during the first $24 \mathrm{~h}$ in the ICU or the last score preceding sedation for intubated/sedated patients, in accordance with the Simplified Acute Physiology Score II (SAPS II) definition [4]), a modified SAPS II score (not including the following: age, presence of chronic comorbidity, GCS score), presence of a significant comorbidity (defined according to SAPS II and the Acute Physiology and Chronic Health Evaluation II [8] criteria), ICH volume in cubic centimeters, midline shift in millimeters, and the presence of IVH.

To adjust for differences in the probability of surgical treatment, we created a propensity score, predicting the likelihood of surgical treatment (propensity score regression adjustment [1]). The propensity score regression model accounts for the patient's age, the hospital, the presence of a significant comorbidity, the ICH location (superficial or deep), the ICH volume (in cubic centimeters), the midline shift in millimeters, and the presence of IVH.

We assessed the performance of the severity of illness model and the propensity score model by calculating their area under the receiver operating characteristic curve (AUC).

We categorized patients into one early ICH surgery cohort and one non-surgical cohort. We defined early ICH surgery as ICH evacuation within 1 day of hospital admission ( $\leq 1$ day). In the primary analysis, patients operated on after the first day ( $>1$ day) of admission were included in the non-surgical cohort. In a secondary analysis, we included all surgically treated patients (early surgery and late surgery) as one group. To minimize the possibility of early mortality rates affecting the results, we conducted a sensitivity analysis, excluding those dying within 3 days ( $\leq 3$ days).

To evaluate cost-effectiveness, we calculated the effective cost per survivor (ECPS) and effective cost per independent survivor (ECPIS). The ECPS and ECPIS are defined as the sum of costs for all patients divided by the number of 1-year survivors or independent 1-year survivors, respectively (e.g., if the sum of costs for 100 patients is $€ 1,000,000$ and there are 20 survivors and 10 independent survivors, the ECPS would be $€ 50,000$ and the ECPIS would be $€ 100,000$ ). We calculated the ECPS and ECPIS separately for the early surgical and non-surgical cohorts.

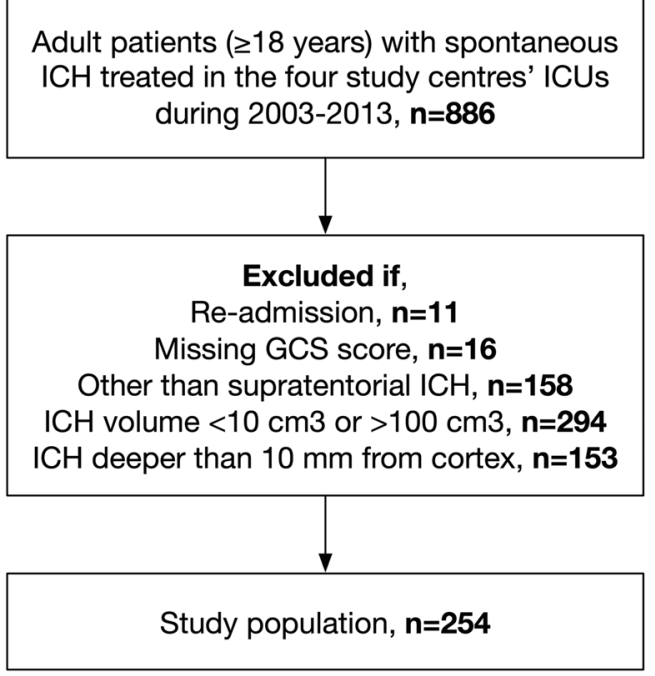

Fig. 1 Flowchart of patient inclusion for this study

The ethics committee of Helsinki University Hospital (HUS 194/13/03/02/2014) and the Finnish National Institute for Health and Welfare (Dnro THL/1298/5.05.00/2019) approved of this registry study and waived the need for informed consent. The research committees of all participating university hospitals approved the study and the data collection. We conducted the study according to the Strengthening the Reporting of Observational Studies in Epidemiology Guidelines (Online Supplement 1) [14].

\section{Results}

\section{Baseline characteristics}

A total of 254 patients were included (Fig. 1). Of these, 68 patients (27\%) were in the early ICH surgery cohort and 186 patients $(73 \%)$ were in the non-surgical cohort. In the nonsurgical cohort, nine patients underwent later ICH surgery (at a median of 3 days, with a range of 2-17 days). Among all patients, $71 \%$ were admitted to the ICU from the emergency department, $9 \%$ from the ward, $6 \%$ from an intermediate care unit, $5 \%$ from the operating theater, and $9 \%$ from other locations.

Patient baseline characteristics are displayed in Table 1. There were no differences in age, sex, presence of severe comorbidity, pre-admission functional status, or median admission year between the early surgical and non-surgical cohorts. Worst 24-h GCS scores for patients in the early surgical cohort were lower than for patients in the non-surgical cohort (a median GCS score of 8 versus 11). Patients in the early surgical cohort had larger ICH volumes (a median of $31 \mathrm{~cm}^{3}$ versus $22 \mathrm{~cm}^{3}$ ). However, there were no differences in the degree of midline shift, depth from the cortex, or presence of IVH. There was no difference in the modified SAPS II score between the groups. Patients in the early surgical cohort had higher levels of treatment intensity. The 
Table 1 Differences in patient characteristics between the surgical cohort and the non-surgical cohort

\begin{tabular}{|c|c|c|c|c|}
\hline & All patients $(N=254)$ & Early surgical cohort $(n=68)$ & Non-surgical cohort $(n=186)$ & $p$ value \\
\hline \multicolumn{5}{|l|}{ Clinical variables } \\
\hline Age (years), median (IQR) & $62(53,71)$ & $65(58,71)$ & $61(51,71)$ & 0.15 \\
\hline$<45$ & $26(10 \%)$ & $4(6 \%)$ & $22(12 \%)$ & 0.21 \\
\hline $45-75$ & $202(80 \%)$ & $59(87 \%)$ & $143(77 \%)$ & \\
\hline$>75$ & $26(10 \%)$ & $5(7 \%)$ & $21(11 \%)$ & \\
\hline \multicolumn{5}{|l|}{ Sex } \\
\hline Female & $97(38 \%)$ & $23(34 \%)$ & $74(40 \%)$ & 0.39 \\
\hline Male & $157(62 \%)$ & $45(66 \%)$ & $112(60 \%)$ & \\
\hline GCS score, median (IQR) & $10(5,14)$ & $8(5,11)$ & $11(5,14)$ & 0.005 \\
\hline $13-15$ & $91(36 \%)$ & $10(15 \%)$ & $81(44 \%)$ & $<0.001$ \\
\hline $9-12$ & $53(21 \%)$ & $21(31 \%)$ & $32(17 \%)$ & \\
\hline $3-8$ & $110(43 \%)$ & $37(54 \%)$ & $73(39 \%)$ & \\
\hline Severe chronic comorbidity & $28(11 \%)$ & $6(9 \%)$ & $22(12 \%)$ & 0.50 \\
\hline Pre-admission antithrombotic medication & $22(9 \%)$ & $9(13 \%)$ & $13(7 \%)$ & 0.12 \\
\hline \multicolumn{5}{|l|}{ Pre-admission functional ability } \\
\hline Independent & $219(88 \%)$ & $57(88 \%)$ & $162(88 \%)$ & 0.86 \\
\hline Dependent & $29(12 \%)$ & $8(12 \%)$ & $21(12 \%)$ & \\
\hline Admission year & $2009(2007,2011)$ & $2009(2007,2011)$ & $2010(2007,2011)$ & 0.47 \\
\hline \multicolumn{5}{|l|}{ Radiological variables } \\
\hline ICH volume $\left(\mathrm{cm}^{3}\right)$, median (IQR) & $25(16,39)$ & $31(23,47)$ & $22(15,37)$ & 0.002 \\
\hline $10-29 \mathrm{~cm}^{3}$ & $157(62 \%)$ & $32(47 \%)$ & $125(76 \%)$ & 0.008 \\
\hline $30-49 \mathrm{~cm}^{3}$ & $59(23 \%)$ & $24(35 \%)$ & $35(20 \%)$ & \\
\hline $50-100 \mathrm{~cm}^{3}$ & $38(15 \%)$ & $12(18 \%)$ & $26(14 \%)$ & \\
\hline Depth from cortex (mm), median (IQR) & $0(0,3)$ & $0(0,2)$ & $0(0,3)$ & 0.18 \\
\hline Midline shift (mm), median (IQR) & $4(0,9)$ & $6(0,9)$ & $3(0,9)$ & 0.10 \\
\hline$\geq 5 \mathrm{~mm}$ & $124(49 \%)$ & $40(59 \%)$ & $84(45 \%)$ & 0.054 \\
\hline Intraventricular hemorrhage & $97(38 \%)$ & $25(37 \%)$ & $72(39 \%)$ & 0.78 \\
\hline \multicolumn{5}{|l|}{ ICU variables } \\
\hline ICP monitoring & $28(11 \%)$ & $12(18 \%)$ & $16(9 \%)$ & 0.042 \\
\hline External ventricular drain & $18(7 \%)$ & $6(9 \%)$ & $12(7 \%)$ & 0.51 \\
\hline Modified SAPS II score*, median (IQR) & $14(8,20)$ & $17(8,22)$ & $14(8,20)$ & 0.30 \\
\hline SAPS II score, median (IQR) & $35(24,51)$ & $40(28,54)$ & $33(23,50)$ & 0.029 \\
\hline TISS-76 mean score per day, median (IQR) & $26(20,32)$ & $29(24,33)$ & $23(17,31)$ & $<0.001$ \\
\hline TISS-76 total score for ICU period, median (IQR) & $63(39,118)$ & $101(57,151)$ & $55(34,101)$ & $<0.001$ \\
\hline \multicolumn{5}{|c|}{ Duration of stay } \\
\hline ICU (days), median (IQR) & $2(1,3)$ & $3(1,4)$ & $2(1,3)$ & $<0.001$ \\
\hline Hospital (days), median (IQR) & $6(3,11)$ & $6(3,9)$ & $6(2,12)$ & 0.72 \\
\hline \multicolumn{5}{|l|}{ Outcome } \\
\hline ICU mortality & $28(11 \%)$ & $0(0 \%)$ & $28(15 \%)$ & 0.001 \\
\hline Hospital mortality & $47(19 \%)$ & $4(6 \%)$ & $43(23 \%)$ & 0.002 \\
\hline 30-day mortality & $76(30 \%)$ & $15(22 \%)$ & $61(33 \%)$ & 0.10 \\
\hline 12-month mortality & $100(39 \%)$ & $20(29 \%)$ & $80(43 \%)$ & 0.050 \\
\hline Alive without a permanent disability & $73(29 \%)$ & $16(24 \%)$ & $57(31 \%)$ & 0.27 \\
\hline
\end{tabular}

Abbreviations: GCS Glasgow Coma Scale, ICP intracranial pressure, ICU intensive care unit, IQR interquartile range, NA not applicable, SAPS II Simplified Acute Physiology Score II, TISS Therapeutic Intervention Scoring System

*SAPS II score without age, GCS, or chronic comorbidities

median ICU stay was slightly longer for those in the early surgical cohort ( 3 versus 2 days), but hospital length of stay was the same (a median of 6 days).

In univariate analysis, the early surgical cohort had lower ICU and hospital mortality rates than the non-surgical cohort (an ICU mortality rate of $0 \%$ versus $15 \%$ and a hospital mortality rate of $6 \%$ versus $23 \%$ ). There were no statistically significant differences in 30-day or 12-month mortality rates between the groups (which were, overall, $30 \%$ and 39\%, respectively). There was no statistically significant difference in the proportion of patients who survived without permanent disability between the groups (which was $29 \%$ overall).

\section{Multivariable analysis}

The severity of illness model performed well in predicting 12month mortality rates (AUC $0.83,95 \%$ CI $0.78-0.88$ ). The severity of illness model performed adequately in predicting the probability of survival without permanent disability (AUC $0.77,95 \%$ CI $0.71-0.84)$. The propensity score regression model for predicting early surgery yielded an AUC of 0.67 (95\% CI 0.59-0.74).

In our primary analysis, when comparing early $\mathrm{ICH}$ surgery to non-surgical treatment, early surgery was associated with a lower 12-month mortality rate (OR 0.22 , 
Fig. 2 Association between surgery and outcome in patients with spontaneous intracranial hemorrhage. An odds ratio lower than 1 indicates a lower risk of death; an odds ratio more than 1 indicates an increased risk of death
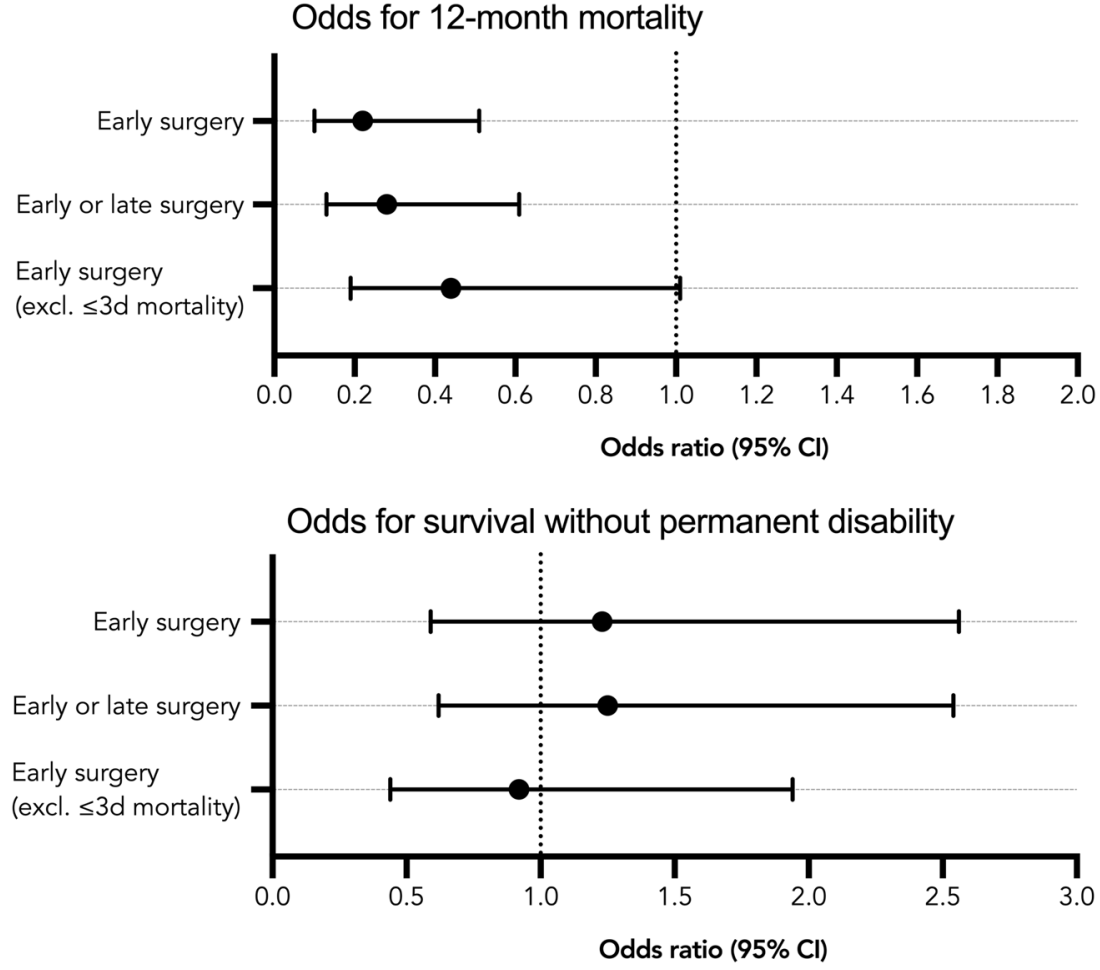

95\% CI 0.10-0.51, $p<0.001$ ) (Fig. 2). There was no association between early ICH surgery and the probability of survival without permanent disability (OR 1.23, 95\% CI $0.59-2.56, p=0.577$ ).

In the secondary analysis, early or late ICH surgery was associated with a lower 12-month mortality rate (OR 0.28 , $95 \%$ CI $0.13-0.61, p=0.001)$, but not with the probability of survival without permanent disability (OR 1.25, 95\% CI $0.62-2.54, p=0.527$ ).

In the sensitivity analysis, after excluding patients who died within 3 days of admission $(n=43)$, the association between early ICH surgery and 12-month mortality rates did not reach statistical significance (OR $0.44,95 \%$ CI $0.19-1.01, p=0.053)$. Further, the sensitivity analysis showed no association between early ICH surgery and the probability of survival without permanent disability (OR 0.92 , 95\% CI 0.44-1.94, $p=0.830$ ).

\section{Cost-effectiveness}

Total 1-year healthcare costs for the early surgical cohort $(n=$ 68 ) were $€ 5,347,630$ and, for the non-surgical cohort ( $n=$ $186), € 8,063,873$. There were 481 -year survivors in the early surgical cohort and 1061 -year survivors in the non-surgical cohort. Thus, the ECPS for the early surgical cohort was $€ 111,409$ (95\% CI €93,551-€129,267), and for the nonsurgical cohort, it was €76,074 (95\% CI €70,081-€82,068). There were 16 patients and 57 patients who survived without permanent disability in the early ICH surgery group and the non-surgical group, respectively. The ECPIS is therefore $€ 334,227$ (95\% CI €316,369-€352,085) for the early surgical cohort and $€ 141,471$ (95\% CI $€ 135,478-147,465)$ for the non-surgical cohort.

\section{Discussion}

\section{Key findings}

In this retrospective, register-based multicenter study of early surgery versus medical treatment in patients with superficial, supratentorial spontaneous $\mathrm{ICH}$, we found that early surgery was associated with lower 12-month mortality rates when compared to non-surgical treatment outcomes. Our results showed that patients undergoing early ICH surgery appeared to be in worse clinical condition than patients who received only medical treatment, but that after adjusting for disease severity and the probability of receiving surgical treatment, early surgical treatment was associated with lower mortality rates. However, early surgical treatment was not associated with an increased probability of survival without permanent disability. Further, surgical treatments led to higher treatment-related costs and were less cost-effective than conservative treatments (resulting in higher ECPS and ECPIS). 


\section{Comparison with previous studies}

The role of surgery in the treatment of spontaneous ICH has been a topic of debate for decades. Despite multiple studies assessing the benefits of ICH surgery, it has remained a controversial treatment option $[10,13,14,23]$. One potential reason for this uncertainty is the heterogeneity of the disease. The location, size, and symptoms of ICH are highly variable, and there is a wide spectrum of differences in patients' medical histories. In clinical practice, one of the main determinants when considering ICH surgery is the location of the hemorrhage. Deep-seated clots are difficult to reach, whereas patients with subcortical hemorrhages are more often offered surgical treatment. Reducing mass effect and alleviating consequent secondary neural damage are considered the main benefits of ICH surgery; removal of the clot may also attenuate the potentially toxic effects that blood byproducts can have and prevent the formation of edema in the surrounding brain tissue $[12,22]$. Interestingly, our propensity score for predicting the probability of early ICH surgery displayed an AUC of only 0.67 , indicating that the indications for $\mathrm{ICH}$ surgery seem to vary in a pseudorandomized manner.

A meta-analysis of 14 randomized controlled trials (RCT) investigating the role of surgery in spontaneous ICHs concluded cautiously that early surgery before deterioration in clinical condition might be beneficial, bearing in mind that this was a very heterogenous group of studies in terms of design, information used, and outcomes [5]. For example, the distance between the hematoma and the cortex was mentioned only in one study. The STICH trial was the first large international, multicenter RCT on early surgery versus best medical treatment in patients with supratentorial spontaneous ICH [13]. The results were neutral, since surgery for superficial lesions improved outcomes while surgery for deeper lesions had the opposite effect. This finding led to another RCT, the STICH II trial, in which the authors selected patients who had benefitted from surgery to be randomized into ICH surgery or best medical treatment [14]. These patients had superficial $(\leq 1 \mathrm{~cm})$ supratentorial ICHs with a volume between 10 and $100 \mathrm{ml}$ (the same as in the present study). STICH II eventually concluded that there was no benefit to early surgery compared to conservative treatment; however, $21 \%$ of the patients initially assigned to the best medical treatment group were operated on later during the study period because of clinical deterioration. Thus, although intention-to-treat analyses were used, the high rate of crossover from the medical to the surgical group may have affected the results.

In this study, we used similar inclusion criteria to STICH II as a means of assessing the potential benefits of surgery in real-life clinical situations. In STICH II, the authors tried to select patients that presented a true clinical equipoise for the attending physicians, whereas our data consist of the results of clinical case-by-case decision-making [14]. It is also worth noting that STICH II defined early surgery as surgery within $12 \mathrm{~h}$ of ictus, whereas we defined it as within $24 \mathrm{~h}$ of admission. Moreover, we did not exclude patients with IVH. All in all, our results were somewhat encouraging, showing a clear association between early ICH surgery and lower 12-month mortality rates in the surgical group versus the non-surgical group. Nevertheless, we found no association between early surgery and the probability of survival without permanent disability, suggesting that ICH surgery may help avoid mortality, but does not improve neurological outcomes. In addition, we performed a secondary analysis that included patients who required delayed surgery. In this secondary analysis, ICH surgery was associated with a reduced 12-month mortality rate when compared to non-surgical treatment. Another recent retrospective study from Finland also found that ICH surgery was associated with lower mortality rates, especially among patients under 70 years old, despite large hematoma volumes. [10]

An interesting finding in our study was that despite patients in the non-surgical group having higher 24-h GCS scores (i.e., being in better clinical condition) significantly lower clot volumes, their in-hospital mortality rate was still significantly higher than that of the early surgery group. Although the baseline variables did not show significant differences between these patient groups, there may still be some hidden confounding factors - especially since this is a retrospective registry study - that led these patients to receive only medical treatment. However, the positive effects of ICH surgery itself may also play a role in explaining this difference.

It is well known that spontaneous ICH is one of the main burdens on healthcare services, incurring high costs partly because patients often need long-term rehabilitation [7]. Compared to subarachnoid hemorrhages (SAH), traumatic brain injuries (TBI), and acute ischemic stroke, spontaneous $\mathrm{ICH}$ is associated with the highest rate of permanent disability and ECPS [17]. Our results showed higher treatment costs for the early surgery group. This may be at least partly due to longer ICU stays and additional costs related to the surgery itself, but it could also be related to the fact that these patients survive and, thus, require prolonged and intensive rehabilitation. It is a self-fulfilling prophecy if clinicians withhold the most aggressive surgical interventions, which may save lives, to severely ill ICH patients, that it will be financially cheaper but possibly unethical. Thus, in assessing healthcare costs, it is preferable to use measures of cost-effectiveness. The most commonly used measure of cost-effectiveness is the cost of a quality adjusted life year (QALY) [21]. Cost-effective treatments are defined as having a QALY of approximately $€ 25,000-€ 35,000$ [11]. Without data on quality of life, costeffectiveness may be quantified as ECPS or, more appropriately, ECPIS. There are no limits to or recommendations for cost-effective treatments in terms of ECPS or ECPIS, and these measures cannot be directly compared to QALY. Still, 
compared to severe TBI (with an ECPS of approximately $€ 80,000$ and an ECPIS of approximately $€ 145,000[16,17])$, SAH (an ECPS of approximately $€ 70,000$, an ECPIS of approximately $€ 95,000$ [17]), acute ischemic stroke (an ECPS of approximately $€ 55,000$, an ECPIS of approximately $€ 105,000$ [17]), and cardiac arrest (an ECPS of approximately $€ 95,000$, an ECPIS of approximately $€ 100,000$ [2]), ICH surgery seems to be cost-effective in terms of survival, with an ECPS of approximately $€ 110,000$, though not in terms of independent living (with an ECPIS of approximately $€ 335,000$ ).

\section{Strengths and limitations}

By using a high-quality, multicenter ICU database like FICC, we were able to collect and analyze prospectively collected data for all patients treated consecutively in participating ICUs. FICC has been extensively validated, and all cases included in the study were also reviewed to confirm diagnoses. In addition, follow-up was comprehensive, and due to the unique landscape of the healthcare system in Finland, we were able to collect full cost-related data for all patients.

Nonetheless, there are some limitations to be considered. First, given the retrospective nature of the study, we can only report associations. Second, we were unable to control for treatment restrictions and withdrawal of care. Ignoring withdrawal of care decisions may affect the results, as it leads to a self-fulfilling prophecy due to withholding treatment as a result of an assumed poor prognosis. To account for this potential source of bias, we conducted a separate secondary analysis that included only those patients who were alive 3 days after admission. This analysis showed a lower 12-month mortality rate for the early surgery cohort; however, this did not quite reach statistical significance (possibly due to lack of power). Third, the FICC database only includes patients treated in the ICU and not in stroke units, where most Finnish ICH patients are treated. Our ICH patients therefore represent a specific subset of patients. Fourth, we used the GCS score as defined by the SAPS II criteria, i.e., the worst GCS score observed during the first $24 \mathrm{~h}$ or the last score before a patient was sedated. In other words, the GCS scores may have been measured at different points for different patients. Fifth, we used admission CT scans and, thus, did not consider the dynamic nature of ICH. For example, we could not verify that the improvement in survival was due to a smaller post-operative ICH volume in operated patients. Sixth, ICH volumes were measured using the $\mathrm{ABC} / 2$ method and not by more modern volumetric methods. Still, this should have minimal effect on our results as the same methods were applied to both groups. Seventh, we used a surrogate marker of permanent disability instead of the more commonly used modified Rankin scale or the Glasgow Outcome Scale. This also means that one should not convert our results directly into these outcome measures.

\section{Conclusion}

Early surgery for superficial, supratentorial spontaneous ICH treated in the ICU may reduce mortality but that it does not affect the probability of survival without permanent disability. However, treatment costs are higher and cost-effectiveness is lower when compared to patients who are treated more conservatively. The role of surgery in spontaneous ICH remains debatable. Further studies are needed in order to identify which ICH patients may benefit from early surgery.

Funding information Open access funding provided by University of Helsinki including Helsinki University Central Hospital. Independent funding support has been received from Helsinki University Hospital (State funding, Finland), Svenska Kulturfonden, Finska Läkaresällskapet, and Medicinska Understödsföreningen Liv \& Hälsa. The funders had no role in the study design, data collection, data analysis, data interpretation, and writing of the manuscript.

\section{Compliance with ethical standards}

Conflict of interest The authors declare that they have no conflict of interest.

Ethics approval and informed consent The ethics committee of Helsinki University Hospital (HUS 194/13/03/02/2014) and the Finnish National Institute for Health and Welfare (Dnro THL/1298/5.05.00/2019) approved of this registry study and waived the need for informed consent. The research committees of all participating university hospitals approved the study and the data collection. We conducted the study according to the Strengthening the Reporting of Observational Studies in Epidemiology Guidelines.

Open Access This article is licensed under a Creative Commons Attribution 4.0 International License, which permits use, sharing, adaptation, distribution and reproduction in any medium or format, as long as you give appropriate credit to the original author(s) and the source, provide a link to the Creative Commons licence, and indicate if changes were made. The images or other third party material in this article are included in the article's Creative Commons licence, unless indicated otherwise in a credit line to the material. If material is not included in the article's Creative Commons licence and your intended use is not permitted by statutory regulation or exceeds the permitted use, you will need to obtain permission directly from the copyright holder. To view a copy of this licence, visit http://creativecommons.org/licenses/by/4.0/.

\section{References}

1. D'Agostino RB (1998) Propensity score methods for bias reduction in the comparison of a treatment to a non-randomized control group. Stat Med 17(19):2265-2281

2. Efendijev I, Folger D, Raj R, Reinikainen M, Pekkarinen PT, Litonius E, Skrifvars MB (2018) Outcomes and healthcareassociated costs one year after intensive care-treated cardiac arrest. Resuscitation 131(Resuscitation 95 2015):128-134

3. Fallenius M, Skrifvars MB, Reinikainen M, Bendel S, Curtze S, Sibolt G, Martinez-Majander N, Raj R (2019) Spontaneous intracerebral hemorrhage. Stroke 50(9):2336-2343 
4. Gall J-RL, Lemeshow S, Saulnier F (1993) A new simplified acute physiology score (SAPS II) based on a European/North American multicenter study. Jama J Am Medical Assoc 270(24):2957

5. Gregson BA, Broderick JP, Auer LM et al (2012) Individual patient data subgroup meta-analysis of surgery for spontaneous supratentorial intracerebral hemorrhage. Stroke J Cereb Circulation 43(6):1496-1504

6. Hemphill JC, Greenberg SM, Anderson CS et al (2015) Guidelines for the management of spontaneous intracerebral hemorrhage. Stroke 46(7):2032-2060

7. Johnson CO, Nguyen M, Collaborators G 2016 S et al (2019) Global, regional, and national burden of stroke, 1990-2016: a systematic analysis for the Global Burden of Disease Study 2016. Lancet Neurol 18(5):439-458

8. Knaus WA, Draper EA, Wagner DP, Zimmerman JE (1985) APACHE II. Crit Care Med 13(10):818-829

9. Kothari RU, Brott T, Broderick JP, Barsan WG, Sauerbeck LR, Zuccarello M, Khoury J (1996) The ABCs of measuring intracerebral hemorrhage volumes. Stroke 27(8):1304-1305

10. Löppönen P, Tetri S, Juvela S, Huhtakangas J, Saloheimo P, Bode MK, Koivukangas J, Hillbom M (2013) A population based study of outcomes after evacuation of primary supratentorial intracerebral hemorrhage. Clin Neurol Neurosur 115(8):1350-1355

11. McCabe C, Claxton K, Culyer AJ (2008) The NICE costeffectiveness threshold: what it is and what that means. Pharmacoeconomics 26(9):733-744

12. Mendelow AD (1993) Mechanisms of ischemic brain damage with intracerebral hemorrhage. Stroke J Cereb Circulation 24(12 Suppl): I115-I117 discussion I118-9

13. Mendelow AD, Gregson BA, Fernandes HM, Murray GD, Teasdale GM, Hope DT, Karimi A, Shaw MDM, Barer DH, investigators for the S (2005) Early surgery versus initial conservative treatment in patients with spontaneous supratentorial intracerebral haematomas in the International Surgical Trial in Intracerebral Haemorrhage (STICH): a randomised trial. Lancet 365(9457): 387-397

14. Mendelow AD, Gregson BA, Rowan EN, Murray GD, Gholkar A, Mitchell PM, Investigators SI (2013) Early surgery versus initial conservative treatment in patients with spontaneous supratentorial lobar intracerebral haematomas (STICH II): a randomised trial. Lancet Lond Engl 382(9890):397-408

15. Meretoja A, Strbian D, Putaala J et al (2012) SMASH-U: a proposal for etiologic classification of intracerebral hemorrhage. Stroke J Cereb Circulation 43(10):2592-2597

16. Raj R, Bendel S, Reinikainen $M$ et al (2018) Temporal trends in healthcare costs and outcome following ICU admission after traumatic brain injury. Crit Care Med 46(4):e302-e309

17. Raj R, Bendel S, Reinikainen M, Hoppu S, Laitio R, Ala-Kokko T, Curtze S, Skrifvars MB (2018) Costs, outcome and costeffectiveness of neurocritical care: a multi-center observational study. Critical Care Lond Engl 22(1):225
18. Reinikainen M, Mussalo P, Hovilehto S, Uusaro A, Varpula T, Kari A, Pettilä V, Consortium FIC (2012) Association of automated data collection and data completeness with outcomes of intensive care. A new customised model for outcome prediction. Acta Anaesth Scand 56(9):1114-1122

19. Steiner T, Salman RA-S, Beer R et al (2014) European Stroke Organisation (ESO) guidelines for the management of spontaneous intracerebral hemorrhage. Int J Stroke 9(7):840-855

20. van Asch CJ, Luitse MJ, Rinkel GJ, van der Tweel I, Algra A, Klijn CJ (2010) Incidence, case fatality, and functional outcome of intracerebral haemorrhage over time, according to age, sex, and ethnic origin: a systematic review and meta-analysis. Lancet Neurol 9(2):167-176

21. Weinstein MC, Torrance G, McGuire A (2009) QALYs: the basics. Value Health 12(s1):S5-S9

22. Xi G, Wagner KR, Keep RF, Hua Y, de Courten-Myers GM, Broderick JP, Brott TG, Hoff JT (1998) Role of blood clot formation on early edema development after experimental intracerebral hemorrhage. Stroke 29(12):2580-2586

23. Zuccarello M, Brott T, Derex L et al (1999) Early surgical treatment for supratentorial intracerebral hemorrhage. Stroke 30(9):1833-1839

\section{Comments}

Supratentorial spontaneous intracerebral hematoma (ICH) is a major source of mortality and morbidity. Although several RCTs on this topic exist, the role early surgical treatment remains debatable. In this study, Luostarinen et al confirms previous RCT findings, with lower mortality but risk of permanent disability in surgically treated cases. Further, Luostarinen et al demonstrates that costs of surgically treated patients are higher, and cost-effectiveness is lower when compared to patients who are treated more conservatively.

This multicenter study using a high-quality Finnish ICU database provides a "real world" setting replicating previous RCT results, supplemented by socio-economic data from the Social Insurance Institute (Kela) in Finland. As such, it provides novel and important insights, while concluding that further studies are needed to identify the potential role of surgery in this patient category.

Jiri Bartek

Stockholm, Sweden

Publisher's note Springer Nature remains neutral with regard to jurisdictional claims in published maps and institutional affiliations. 